\title{
EL EMPRESARIO EN EL ANÁLISIS ECONÓMICO, CARACTERÍSTICAS Y FUNCIONES.
}

\section{THE LEADING ROLE OF AN ENTREPENEUR}

\begin{abstract}
María Teresa Buitrago. Departamento de Administración. Grupo de Investigación en Empresariado. J aime Alberto Valencia Ramos. Departamento de Economía. Grupo Desarrollo y Globalización. Participantes: German Rojas Arias. Juan Carlos Marín Sánchez. Asistentes de Investigación: Olga Lucia Cardona. Luis Felipe Ramírez Henker.
\end{abstract}

Palabras claves: Empresario, organización, neoinsitucionalismo, costos de transacción, sistema de precios, sistema de mercado, empresa.

Keywords: Entrepreneur, organization, neo-institutionalism, transaction costs, price system, market system, company.

\section{Resumen}

Para la teoría clásica y neoclásica de economía el empresario fue considerado fundamentalmente un factor de producción, que jugaba un papel pasivo y en algunos casos de coordinación en el sistema de precios, para la corriente neoinstitucionalista el empresario es un agente activo que toma decisiones, dinamiza la organización y el sistema de precio, por cuanto es coordinador que busca resolver los problemas que plantea la economía, referentes a la asignación de recursos al interior de las organizaciones.

\section{Abstract}

In the classical economic theory and neoclassical theory, an entrepreneur is basically considered a production factor, who had played a passive role or, in some cases, if any, a coordinator of the price system. However, in the neo-institutionalism, an entrepreneur is an active agent, who makes decisions and pushes forward the organization and the price system. Finally, the entrepreneur tries to solve the problems imposed by the economy, referring to the allocation of company resources ${ }^{1}$.
1 Resúmenes traducidos por el gestor de traducciones del Instituto de Idiomas de la UAM, Carlos A. Muñoz Torres, Máster en Teoría de la Traducción, Universitat Autònoma de Barcelona.

Resúmenes revisados por Kevin Guzzo, nativo del inglés, estudiante de la Maestría en Traducción de la Universidad Autónoma de Manizales.

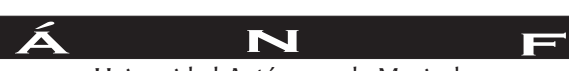
Universidad Autónoma de Manizales

\section{$\mathbf{R}$}

Año 15 Número 25,julio-diciembre de 2008 


\section{Introducción}

El presente documento, pretende mostrar la evolución del concepto de empresario en la teoría económica a partir de la escuela clásica, haciendo el tránsito por la Neoclásica, hasta llegar a las concepciones Neo institucionalistas imperantes en la actualidad. Cabe resaltar, que a través de las diferentes visiones históricas, se le ha concedido al empresario un papel protagónico en el devenir del sistema de mercado, contextualizándolo en el momento histórico y ajustándose a las teorías económicas imperantes.

Las diferentes concepciones sobre "el empresario" a través de la historia de la teoría económica, sientan las bases para un área investigativa referente a los temas de dicho agente, en cuanto a sus características, comportamientos, roles e incidencia en la vida económica.

Las concepciones sobre "empresario" se abordaron sobre las diferentes corrientes y autores que marcando diferencias han sido las más representativas del pensamiento capitalista. Las diferentes perspectivas han lleva todas a resaltar el papel del empresario como agente dinamizador y transformador en un sistema económico de mercado, así mismo permite determinar las competencias necesarios que un Economista Empresarial debe desarrollar con el fin de dar solución a los problemas empresariales desde una perspectiva económica dándose un valor agregado al lo que tradicionalmente ha sido el campo de acción del economista, y respondiendo a las necesidades del medio en procura del cumplimiento de la misión institucional.

\section{La metodología}

Para abordar el tema de la investigación "El empresario en el Análisis económico", se acudió a la indagación de fuentes primarias y secundarias de autores, con el fin de identificar la concepción de empresario y su papel en el sistema de mercado, visto desde las distintas corrientes del pensamiento económico.

Se desarrollaron en la investigación los siguientes momentos:

1. Se estableció la taxonomía de escuelas económicas y de autores más relevantes dentro de cada una de ellas, de acuerdo a lo que tradicionalmente han aportado al concepto de empresario y la relación de sus fundamentos con el sistema económico capitalista, en el contexto histórico pertinente.

2. Análisis y contraste de los autores relevantes en cada escuela.

3. A partir de la lectura de autores se infiere el concepto de empresario, el papel que juega y la importancia que representa para la escuela respectiva.

\section{Escuelas y autores referenciados:}

1. ESCUELA CLASICA: Adam Smith, J uan Bautista Say, J hon Stuart Mill.

2. ESCUELA NEOCLASICA: Walrras, Alfred Marshall, Keynes.

3. FASE DE TRANSICION: Shumpeter, Von Hayek, Galbraith, Kirzner.

4. ESCUELA NEOI NTITUCIONAL: Ronald Coase, Williamson.

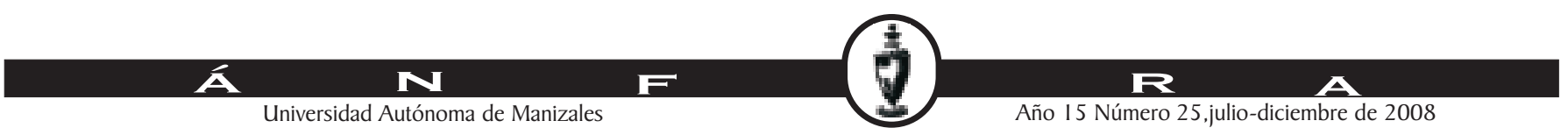




\section{Escuela clásica}

En el contexto de la revolución industrial, el empresario, para esta escuela de pensamiento, fue considerado como el capitalista o director de industria, que recibía una contribución, denominada beneficios, por otorgar su capacidad productiva al sistema económico.

El capitalista, como grupo social, está asociado a la acumulación de bienes de capital, que como lo argumenta Smith, la acumulación de capital sólo es necesario en las sociedades avanzadas, las cuales se caracterizan por la división del trabajo y el intercambio de productos; se presenta así un círculo virtuoso que indica que a mayor acumulación de capital, mayor división del trabajo y mayor intercambio, que a su vez genera mayor acumulación de capital. Todo lo anterior es propio de un sistema económico capitalista.

En el mismo sentido, argumenta Smith, con respecto a la retribución al capitalista, “EI beneficio es tan fluctuante que ni siquiera la persona ocupada en un negocio concreto puede estar siempre segura de cuál es su beneficio anual medio. Le influyen no solo cualquier variación de los precios de las mercancías de las que se trate, sino la mejor o peor fortuna de sus competidores, de sus clientes y los miles de accidentes que pueden acaecer a las mercancías cuando éstas se transportan por mar, por tierra, o incluso cuando están almacenadas. Por tanto el beneficio varia no sólo de año en año, si no de día en día, incluso casi de hora en hora. Por ello precisar el beneficio medio de todos los sectores de un gran reino es mucho más difícil, y juzgar lo que ha podido suceder previamente o en periodos remotos de tiempo, con algún grado de precisión, es totalmente imposible." (Smith, 1981).

Para Smith el empresario no es diferente del capitalista, el cual juega un papel importante en el modelo económico, su tarea consiste en ahorrar y vigilar el adecuado funcionamiento de las empresas.

El capitalista es un acumulador de capital y esa es su tarea principal, asigna el capital en diferentes inversiones, que amarrado a su propiedad la administra buscando el mayor benéfico personal. Para Adam Smith el empresario es un capitalista activo ( gerente propietario) que hace uso del capital para contratar pocos trabajadores.

Juan Bautista Say:

Con este autor, se muestra una diferencia clara entre el concepto de capitalista y de empresario, lo cual constituye un avance significativo, con respecto a la concepción del empresario en la escuela clásica, el empresario pasa de ser un factor más de producción a un combinador y administrador de factores productivos.

El capitalista aporta capital y por ello se le paga un interés que es el costo del servicio de prestar su capital para la producción. El empresario es aquel que compra servicios productivos para transformarlos en artículos nuevos y luego venderlos, su retribución se denomina beneficio que consiste en la diferencia entre precio de venta y costo de producción.

\section{ㅅ․}


Las funciones del empresario se enmarcan en:

1. Conocimiento de los procesos naturales. Científico

2. Aplicación de estos conocimientos a un fin práctico. Empresario

3. Esfuerzo para llevarlo a cabo. Trabajador.

En palabras de Say, "Para obtener cualquier tipo de producto primero hubo que estudiar el progreso y las leyes de la naturaleza respecto a él, ¿Cómo se habría fabricado una cerradura, si no se hubiera logrado conocer las propiedades del hierro, y por que medios se le puede extraer de la mina, refinarlo, ablandarlo y darle forma? Después hubo que aplicar esos conocimientos a un empleo útil: I maginar que al dar forma al hierro de cierta manera se obtendría un producto que tendría cierto valor para los hombres. Finalmente, hubo que llevar a cabo el trabajo manual indicado por las dos operaciones anteriores, es decir, forjar y limar las diferentes piezas de las que consta la cerradura." (Say, 2001).

Como agente principal de la producción, es el empresario quien organiza, coordina, intermedia entre la oferta y la demanda, asumiendo el riesgo, pues está comprometiendo recursos (fortuna y honor) al aportar su capital, por tanto recibe remuneración compuesta de los siguientes elementos:

El interés que se paga al capital aportado.

El pago del salario de su propio trabajo.

Un premio por asumir riesgo.

La asunción del riesgo, lleva a Say a diferenciar entre capitalista activo y capitalista rentista, son los primeros quienes toman decisiones que gobiernan la vida económica, y por ello pasa a tener más importancia que la de capitalista rentista.

\section{John Satuart Mill}

Para MILL, los factores de producción son fundamentalmente, el trabajo, la tierra (agentes naturales) y los productos del trabajo previamente acumulados (capital), estos proveen al trabajador, en una sociedad no primitiva, de las herramientas necesarias para adelantar la labor, como son el refugio, el alimento, las materias primas; es decir, el capital permite al trabajador mantenerse y satisfacer necesidades mientras llega el momento del retorno de la producción. (Mill, 1941)

El capital surge de un proceso de ahorro, diferencia entre ingresos y gastos, la generación de excedente de ingresos sobre los gastos permite aumentos del ahorro y con ello del capital, "Si todas las personas gastaran todo lo que producen, y toda la renta que perciben de lo que es producido por otros, en goces personales, el capital no podría aumentar" (Mill, 1941).

Lo anterior plantearía que al interior del sistema de mercado, basado en la propiedad privada, debe existir un agente promotor de la generación de capital, caracterizado por su frugalidad, bien sea por sus altos ingresos o bajos consumos, es decir un agente (capitalista) que se sacrifica hoy para obtener mayores beneficios al futuro, se puede decir que el capital, es la materialización de una decisión intertemporal de llevar consumo presente hacia consumo futuro.

\section{A N N Universidad Autónoma de Manizales




\section{Escuela neoclásica:}

Leon Wallras:

En este autor, el concepto de empresario, se analiza a la luz de lo que él denominó "Equilibrio General".

Con Wallras, el concepto de equilibrio, se basa en su teoría de "Equilibrio General", en la cual muestra la manera como, a partir de intercambios voluntarios entre individuos informados, interesados, individualistas, racionales que buscan la mejor opción, llevan a una empresa a tomar decisiones en cuanto a producción y distribución de la renta, que daba como resultado una distribución eficiente y beneficiosa para oferentes y demandantes.

En el concepto de equilibrio, es importante especificar dos elementos, el primero, el intercambio voluntario que se da en el mercado, conceptualmente es la forma única de interacción social aceptada, en el sentido que este intercambio saca del modelo la presión que pueden ejercer los grupos de presión u otro elemento desestabilizador de un competencia perfecta.

El segundo elemento, las decisiones de las empresas que obliga al empresario a tomarlas con respecto al cálculo o previsión de:

Las cantidades necesarias de materia prima que requiere para desarrollar el proceso productivo, el número de unidades a fabricar y los precios, estos últimos se calculan teniendo como base los precios de los bienes de consumo, intermedios, el costo de los servicios por el uso de los bienes de capital y el costo del dinero, para Walras la utilidad del empresario está dada por la rentabilidad generada por el uso de los factores de producción de los cuales éste es propietario.

En el proceso de elaborar los pronósticos, el empresario, individualmente considerado, mide y estima, no solo sus costos objetivos de producción, sino también los comportamientos esperados de otros empresarios. Esto se traduce en decisiones estratégicas.

Lo anterior corresponde a la incorporación del empresario como cuarto factor de producción, como bien argumenta Walras: "Llamamos terrateniente a cualquier poseedor de tierras, trabajador al poseedor de facultades personales, capitalista al poseedor de bienes de capital. Y hora llamaremos empresario a una cuarta figura, totalmente distinta de las anteriores, cuyo papel consiste en tomar en arriendo la tierra del terrateniente, las facultades personales del trabajador y los bienes de capital del capitalista, para combinar los tres servicios".

Alfred Marshall: (1842-1924)

En relación con el papel del empresario en el mercado, se hace referencia al concepto de los negocios propuesta por Marshall, quien argumenta; "la organización de los negocios, se llame empresario o "factor organización": insiste en que no es el capital quien contrata al trabajo, si no la "capacidad para los negocios que dispone de capital" (Santos R, 1997).

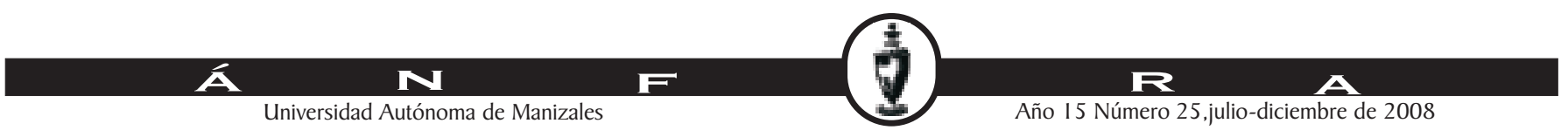


Como bien se infiere a partir del anterior argumento, no es solamente la cantidad de capital y trabajo la que determina el nivel de producción o riqueza, como lo definiera la perspectiva neoclásica en su función de producción tradicional, por tanto, entra al modelo el cuarto factor de producción, el empresario, que mediante la organización y combinación de los factores de producción, genera aumentos en el nivel de riqueza.

Para Marshall "la innovación y el progreso a largo plazo" son los dos aspectos más importante de la economía. La innovación se amarra a la organización en un marco estático hipotético, de allí se define la función empresarial, que para este autor, era un empresario-administrador, él afirma que la dirección de los negocios requiere de una persona con sentido común y conocimientos generales, "Ios conocimientos técnicos y la pericia se hacen cada día menos importante en relación con las amplias y no especiales facultades de buen juicio, rapidez, inteligencia, previsión y perseverancia de fines “(Santos R, 1997).

En este sentido, se concibe al empresario como un "capitalista polifacético" que a partir de un capital invertido logra rendimientos constituidos por los "fondos de comercio" y su gestión, este empresario también puede obtener "ganancias extraordinarias" (Cuasi-renta) por lograr colocar mayores valores a algunos activos.

Los beneficios extraordinarios los alcanza el empresario que tiene unas características excepcionales para hacer los negocios y puede desarrollar técnicas mejores que sus rivales y logre mejores beneficios que ellos, es decir, que tome decisiones estratégicas. El empresario también puede lograr beneficios a partir de la especulación pero considera que no es esta la actividad principal.

Las retribuciones para la capacidad organizacional del capital - talento empresarial, es el resultado de un precio que se forma a partir de otros precios; como lo define Marshall; "la oferta de capacidad para los negocios que dispone de capital se acomoda en general a la demanda $y$, por consiguiente, tiene un precio de oferta bastante bien definido.....constituido por tres elementos: el primero es el precio de oferta del capital; el segundo, el precio de oferta de la capacidad y energía para los negocios; y el tercero, el precio de oferta de aquella organización mediante la cual la aptitud comercial adecuada y el capital necesario son puestos en contacto" (Santos R, 1997).

La retribución a la capacidad organizativa del capital, el empresario, está determinada por:

\footnotetext{
El tamaño de la organización, determinada por la cantidad de capital.

La cantidad de capital variable (mano de obra).

El grado de dificultad que implica la organización.

La necesidad de innovación.

El nivel de riesgos que se asume.

La cantidad de trabajo de dirección.

El grado de especulación de la industria.
}

En este sentido, en la medida en que la dificultad de organizar el capital o generar empresa sea mayor, este deberá tener una mayor retribución; como bien argumentaba Marshall, "el capital irá hacia los más capaces", es así como, un hombre que posee capacidades excepcionales para los negocios, no necesariamente tiene que ser dueño del capital, puesto que puede ocupar un alto cargo, tanto en el sector público como privado.

\section{A N} Universidad Autónoma de Manizales 
Según la teoría redistributiva del ingreso, la existencia de ricos y pobres se debe a las dotaciones naturales - capacidades y aptitudes - de los hombres, más que a su origen económico, por tanto el papel del Estado se reduce a respetar estas condiciones o a potencializar las capacidades.

La retribución al empresario, que depende de los factores anteriormente señalados, adquiere una dinámica de expansión determinada por la innovación, entendida como, "salirse de lo corriente", ella va configurando la organización industrial, la cual es la relación entre empresas y empresarios, que se desarrolla en un entorno regulado por restricciones formales (legales) e informales (pactos de caballeros), la característica de dichas relaciones conduce a la creación de empresas por acciones y grandes corporaciones que configuran monopolios y mayores retribuciones.

No obstante, el argumento anterior, las tendencias actuales son diferentes, en el sentido que en los últimos años la creación de empresas a nivel mundial se ha dado más en el sector de la pequeña y mediana empresa que en el de la gran empresa, así como en sectores de servicio y de mayor tecnología, que incentivan la unipersonalidad empresarial y el autoempleo.

\section{OTROS AUTORES POSTERIORES A LOS NEOCLÁSICOS:}

J oseph Schumpeter (1883-1950):

El empresario en una sociedad capitalista juega un papel fundamental; no es el inventor, ni quien decide arriesgarse con su capital en una aventura empresarial; concibe la ciencia económica como una teoría de la acción más que de la decisión, acción humana a lo largo de cuyo proceso se producen una serie de interacciones y procesos de coordinación, dependiendo de la perspicacia mostrada en el ejercicio de la acción empresarial, por parte de los diversos actores implicados.

Para Schumpeter, el empresario es la fuente del cambio que decide y administra el curso a seguir; entonces, el espíritu empresarial pasa a ser un importantísimo factor de producción y un motor del crecimiento económico, que a su vez, propicia el proceso capitalista de la acumulación de capital, y por otra parte, genera desarrollo económico a través del proceso de innovación; de Wallras tomó la noción de empresario, pero en lugar de su figura pasiva en el sistema del "equilibrio general", la sustituye por un agente activo del progreso económico.

Schumpeter introduce, en su propuesta de desarrollo económico, dos conceptos: la innovación como causa del desarrollo, anotado anteriormente, y el empresario innovador como propiciador de los procesos de innovación.

Schumpeter, define la innovación por medio de la función de producción, entendida ésta como "la manera en que varía la cantidad del producto si varían las cantidades de los factores". Si en lugar de variar las cantidades de factores, variamos la forma de la función, tendremos una innovación; son transformaciones para nuevos productos; la innovación es un acto de la voluntad más que del conocimiento, donde ocupan un lugar preponderante, los estímulos. En este sentido, llamaríamos "empresa" a la realización de nuevas combinaciones, y "empresarios", a los encargados de dirigir dicha realización.

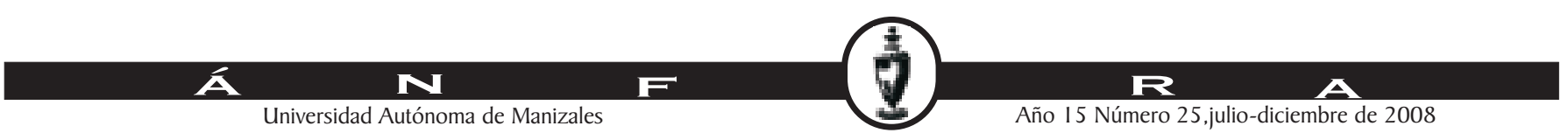


Para Schumpeter se da una rotación empresarial a través de lo que él denomina "proceso de destrucción creativa", esto es, un proceso donde la generación de nuevas empresas (más eficientes) necesariamente trae consigo la salida de las empresas existentes menos eficientes; y complementando esta formulación, Kaldor, anota que el dinamismo del sistema se centra en el efecto que la intensificación de capital tiene sobre la productividad vía la función de progreso técnico, efecto que depende del dinamismo empresarial en incorporar nuevas tecnologías (Chica, 2005).

Ante la realización de una innovación, Schumpeter destaca que el empresario deberá vencer tres dificultades:

- La incertidumbre,

- La conciencia del hombre de negocios ante la resistencia de la gente por realizar algo nuevo, y

- El medio social que se puede manifestar hostil ante las innovaciones.

Schumpeter describió la innovación de varias maneras. Primero enumeró los tipos de nuevas combinaciones que subyacen en el desarrollo económico. Comprenden las siguientes:

- La introducción de un nuevo bien o de una nueva calidad de un bien,

- La introducción de un nuevo método de producción,

- La apertura de un nuevo mercado,

- La conquista de una nueva fuente de aprovisionamiento, y

- La creación de una nueva organización de cualquier industria (por ejemplo, la creación o destrucción de un monopolio).

Friedrich Von Hayek (1889-1992):

El empresario es un elemento importante en el funcionamiento del mercado ya que siempre está alerta para explotar las oportunidades que éste ofrece; siempre responde a los cambios y, como nadie posee todo el conocimiento, los empresarios exitosos serán aquellos que logren aprovechar las oportunidades que entrega el estar en un mercado donde la información es imperfecta.

"Cada uno de nosotros posee una pequeña fracción de todo el conocimiento y la información disponible en el mundo y, sin embargo, de algún modo todas nuestras actividades interdependientes deben ser coordinadas para que resultemos beneficiados con la especialización y experiencia de nuestros congéneres".

Israel m. Kirzner (1930- ):

“La función empresarial en el mercado es difícil de comprender. Lo demuestra la eliminación virtual de dicho papel en las exposiciones más recientes de las teorías de los precios, así como en los múltiples y cuidadosos intentos de autores anteriores para definir al empresario y distinguir su papel del capitalista o el empleado dirigente. Estos intentos reflejan el deseo de identificar con precisión algo cuya presencia se siente indudablemente pero que, superficialmente, sólo se presta a una definición vaga. A mi modo de ver, es posible aferrar ese elemento esquivo de la empresarialidad de una manera satisfactoria. Además, creo que es de la mayor importancia conseguirlo para comprender el proceso del mercado. Una de las distinciones entre la teoría del mercado

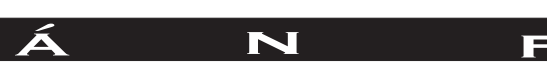

Universidad Autónoma de Manizales 
aquí definida y la que predomina en los textos sobre teoría de los precios hoy en día es que esta última carece de una apreciación adecuada de la naturaleza y función de la empresarialidad en el sistema del mercado" (Santos R, 1997).

Al igual que Mises, Kirzner plantea que el concepto de mercado en donde se realiza la acción humana del empresario, es más que una mera eficiencia del manejo de recursos productivos, y más que un conjunto interactuante de agentes que toman decisiones "economizando y maximizando sobre una serie dada de fines y medios"; es realmente la acción y la decisión de seres humanos comprometidos en la "acción humana" (Mises), elemento consubstancial al empresario, autor de decisiones.

"En toda economía real y viva, todo actor es siempre un empresario", y su papel consistirá en estar atento a descubrir y aprovechar la información que proviene de oportunidades, a pesar, o mejor para bien, de poseer información imperfecta le permite toma de decisiones en función de la empresarialidad, ya que la plena información de los atributos del mercado, nos devolvería al "equilibrio general", (Walras) Ilevando al empresario a un papel reduccionista de carácter meramente economicista.

En cuanto al papel del "empresario" en su función de producción, Kirzner anota que ésta se cumple fundamentalmente en la conjunción imperfecta de los mercados de factores y el de los productos (deficiente coordinación), involucrando un problema de transacción, afectando los precios, la información y la misma eficiencia de los mercados.

\section{El empresario en la perspectiva neoinstitucional:}

Ronald Coase: (1910-)

Se plantea la pregunta: ¿En qué casos cumple el empresario una función de coordinador?

A partir de los postulados neoclásicos, Coase, profundizó y reformo la estructura de pensamiento económico imperante, al considerar que el empresario es una agente activo coordinador, que puede llegar a reemplazar el sistema de precios al interior de las empresas; en efecto, al considerar la empresa no simplemente como una caja negra, este autor argumentaba que "el empresario se ocupa de la división del trabajo dentro de cada empresa, y planifica y organiza con gran minuciosidad, pero él está vinculado con la amplia especialización económica de la que el mismo es una unidad especializada" (Coase, 1936).

Con esto pretende Coase, mostrar que en el sistema de mercado capitalista, existen dos mecanismos coordinadores, uno que es el propio sistema de precios, que es exógeno a la organización - empresa, y otro que funciona al interior de la organización, el empresario; en este caso, el empresario toma decisiones de asignación, distribución de recursos al interior de la empresa, pero considerando las condiciones externas y los resultados de la autorregulación del mercado.

La existencia de costos de transacción presentes en el intercambio al interior del sistema de precios, asociado a los costos de organización, es un postulado ausente en el análisis neoclásico, que da lugar a fallas de mercado e imposibilitan el logro de la eficiencia; es en este sentido, que se configura la organización de la empresa como mecanismo alternativo para la disminución de los costos de transacción.

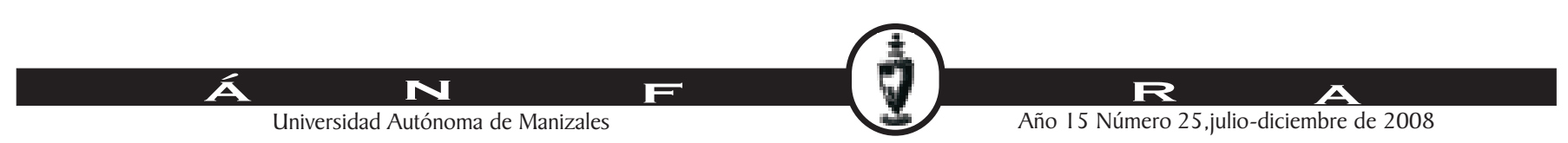


Eggertsson, propone los factores explicativos de los costos de transacción, propio del intercambio en un sistema de mercado ( Santos R, 1997):

- La Búsqueda de Información sobre, precios, cantidades, vendedores, compradores, preferencia y gustos.

- $\quad$ El proceso y duración de la negociación entre los agentes participantes en el mercado.

- $\quad$ La elaboración, aplicación y aceptación de los contratos.

- La vigilancia y control de los compromisos asumidos en los contratos.

- $\quad$ Protección y determinación de los derechos de propiedad.

Oliver e. Williamson

La transacción es la unidad de análisis básica en el modelo económico de "costos de transacción"; la empresa busca organizar las transacciones a menores costos de los que se logran en el mercado; en este sentido, el tamaño de la empresa y sus expansiones están en relación directa con la diferencia positiva entre los beneficios adicionales y los costos adicionales.

El análisis de la transacción llevó a Williamson a plantear que el estudio de la organización o la empresa deben ocupar una lugar destacado en el análisis económico, mediante el modelaje del comportamiento de las organizaciones, en este sentido, los economistas se deben tomar en serio el estudio de la organización.

Los costos de transacción son un campo investigativo en la medida en que al pertenecer a la corriente institucionalista se hace más empírico el fenómeno y formulan respuestas nuevas a los interrogantes tradicionales de la economía, en relación a la asignación de recursos y su grado de utilización; se plantean también preguntas como ¿por qué surgen las instituciones?. ¿Cómo se aplica el razonamiento micro?.

La economía de los costos de transacción. Tiene 3 sentidos básicos

- Supuestos conductistas. Es el supuesto cognoscitivo que postula que los agentes humanos son racionales con limitaciones (Racionalidad limitada).

Los agentes humanos propenden al oportunismo, se refiere a la búsqueda del interés propio que se formalizan a través de relaciones que conllevan un costo asociado a las garantías y a los beneficios de quienes participan.

Las organizaciones son los instrumentos que permiten el logro de los objetivos humanos que de forma individual serían más costos, por cuanto los seres humanos están limitados en aspectos como conocimiento, previsión.

Es por eso que en el modelo de Williamson, la firma es una forma de organización económica relevante, en la que se expresan de manera clara los problemas asociados a los costos de transacción, proceso de toma de decisiones, teniendo en cuenta que las empresas enfrentan un entorno complejo: muchos mercados, varios competidores, dispersión de precios, variedad de calidades, etc. Toma decisiones con fuerte incertidumbre económica, esta se da porque los agentes no conocen toda la información relevante para tomar decisiones óptimas que permitan maximizar beneficios. (Ayala, 2000).

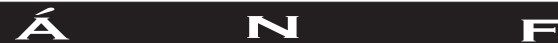

Universidad Autónoma de Manizales 


\section{Conclusiones}

- El concepto de empresario a lo largo de la historia siempre ha estado presente en la teoría económica, en ciertos momentos se asumió como un factor de producción, posteriormente se le considera un agente coordinador hasta el punto de llegar a ser entendido como un dinamizador del sistema al interior de la organización y del mercado.

- En la perspectiva del pensamiento económico la empresa fue considerada como una "Caja Cerrada" o una función de producción, no obstante, adquiere su propia dinámica y relevancia en la teoría económica de principios del siglo XX en la medida en que, la empresa existe por la existencia del empresario, es decir, es este agente quien determina la existencia de la empresa.

- La organización o empresa es el reflejo de las decisiones del empresario en un contexto de información asimétrica, riesgo e incertidumbre.

- Para el análisis económico es relevante más que el concepto de empresa, la actividad que desarrolla el agente denominado empresario quien da vida a la organización.

- El empresario es un agente polifacético que toma decisiones en contextos inciertos tanto en organizaciones privadas como públicas, buscando optimizar la asignación y eficiencia de recursos al interior de éstas.

- El concepto empresario no solamente compete a la disciplina administración como cuerpo de teorías o modelos de comportamiento, como tradicionalmente se ha pensado por parte de cientistas sociales, sino que también el concepto, características y comportamiento del empresario es modelado por otras disciplinas como la sociología, la sicología y de manera relevante por la economía.

- El estudio de la toma de decisiones por parte del empresario, las formas de organización que adopta y los impactos de ello en el sistema de precios se constituyen en un área de investigación de importancia teórica y empírica para la economía a partir de los postulados neoinstitucionalistas, esto hace de esta área un campo amplio de investigación económica interdisciplinaria.

- La teoría neoinstitucionalista y sus conceptos sobre empresario y organización posibilitan el desarrollo de la economía empresarial como especialidad de la ciencia económica.

- En estas indagaciones se puede apreciar la importancia que juega el empresario dentro del sistema económico de mercado, la necesidad de estudiarlo permanentemente con el fin de determinar las competencias necesarias para su formación y dar respuesta a las exigencias del entorno.

- El campo de acción de un economista tradicionalmente ha estado limitado a los aspectos macroeconómicos, sin embargo, a partir de la presente investigación se puede afirmar que el tema empresarial y organizacional es un alternativa de estudio con un enfoque microeconómico para la ciencia económica y en la formación del economista de empresa. 


\section{Bibliografía}

SMITH, Adam . La Riqueza de las Naciones. Edit. Fondo de Cultura Económica 1981. AYALA ESPINO, José. Instituciones y economía una introducción al neo institucionalismo económico. Edit. Fondo de Cultura Económica

SANTOS REDONDO, Manuel, Empresa y Empresario en la Historia del Pensamiento Económico. Alianza Editorial. 1997 Madrid

COASE, Ronald. La Naturaleza de la Empresa. 1937.

MILL, J ohn Stuart Principios de Economía Política. Fondo de Cultura Económica. 1964.

EKELUND Robert B. - HEBERT Robert F. Historia de la Teoría Económica y de su Método. Tercera edición 1999 Mc Graw Hill.

RICARDO, David. Principios de economía Política y Tributación. Primera edición 1959 Fondo de Cultura Económica.

SAY, J ean Bautista. Economía Política. Editorial fondo de Cultura Económica.

SHUMPETER, J osphe. Teoría del desenvolvimiento económico. Edit. Fondo de Cultura Económica.

GALBRAITH, J.K. , El nuevo estado industrial. Biblioteca de la empresa. Tercera edición. Septiembre 1985. Editorial Orby.

WI LLI AMSON, Olivwe E. y WINTER, Sidney G. La Naturaleza de la empresa. Origen evolución y desarrollo. Fondo de cultura económica. México 1996.

SHUMPETER, J.A. Capitalismo socialismo y democracia. Editorial Orby. 1983.

ROLL, Eric . Historia de las doctrinas económicas. Fondo de cultura económica. Edición tercera edición 2002.

SCHEIFLER AMÉZAGA, Xavier. Historia del pensamiento económico. Editorial trillas. México 1979. Cuarta edición 1979.

KALECKI, Michael. Ensayos escogidos sobre dinámica de la economía capitalista. Fondo de cultura económica. Primera edición 1977.

HERRERIAS, Armando. Fundamentos para la historia del pensamiento económico. Editorial Limusa. Quinta edición 2005.

KEYNES, J.M. Teoría general de la ocupación el interés y el dinero. Fondo de cultura económica. Segunda edición 1986.

HANSEN, Alvin H. Guía de Keynes. Fondo de cultura económica. México. Primera edición 1978.

SHUMPETER, J.A. Sintesis de la evolución de la ciencia económica y su método. Editorial Oikos. Barcelona segunda edición 1967.

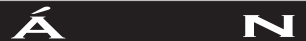

Universidad Autónoma de Manizales 